\title{
Doppler sonography of the uterine and the cubital arteries in normal pregnancies, preeclampsia and intrauterine growth restriction: evidence for a systemic vessel involvement
}

\author{
Barbara Schiess|1,*, Franz Kainer', Renate \\ Oberhoffer $^{2}$, Katharina Jundt ${ }^{1}$ and Klaus Friese ${ }^{1}$ \\ 1 Department of Obstetrics and Gynecology, Prenatal \\ Medicine Unit, Ludwig-Maximilians University Munich, \\ Germany \\ 2 Pediatric Cardiology Unit, German Heart Center, \\ Technical University Munich, Germany
}

\begin{abstract}
Aims: The decrease in uterine resistance during normal pregnancy is known to be related to invading trophoblast cells which derive from placental tissue. Uterine and peripheral resistance is elevated in preeclampsia. The aim of the present study was to prospectively examine uterine and peripheral resistance in pregnancies complicated by preeclampsia (PE), fetal intrauterine growth restriction (IUGR) and pregnancy induced hypertension (PIH).

Methods: Sixty-seven women with normal pregnancies, 17 with PE, 12 with IUGR underwent Doppler sonographic investigation of the uterine and the cubital arteries. The Pulsatility Index (PI) was calculated for each vessel. Statistical analysis was performed and a P-value $<0.05$ was considered significant.

Results: Patients with preeclampsia and IUGR showed a significant higher resistance at the placental (mean PI 1.267 and 1.063), nonplacental (mean PI 1.631 and 1.124) and cubital artery (mean PI 3,777 and 3.995) compared to the normal pregnancy group (mean PI 0.678; 0.859 and 2.95 respectively). Mean birth weight in the PE group was $1409 \mathrm{~g}$, in the IUGR group $1649 \mathrm{~g}$ and $3419 \mathrm{~g}$ in the normal pregnancy group.

Conclusions: Pregnancies with IUGR are associated with elevated peripheral resistance in the maternal arterial system as seen in pregnancies with preeclampsia. Our findings encourage to further investigate the maternal vascular system in high risk pregnancies.
\end{abstract}

\footnotetext{
${ }^{*}$ Corresponding author:

Dr. Barbara Schiessl

Maternal Fetal Medicine Unit

1st Department of Obstetrics and Gynecology

Ludwig-Maximilians-University Munich

Maistr. 1180337 Munich

Germany

Tel.: + $00498951604111 / 4172$

Fax: +00498951604913

E-mail: Barbara.Schiess।@med.uni-muenchen.de
}

Keywords: Doppler sonography; intrauterine growth restriction (IUGR); pregnancy; preeclampsia; pregnancy induced hypertension.

\section{Introduction}

During pregnancy the spiral arteries, which derive from the left and right uterine arteries, undergo a transformation resulting in low resistance. Until the end of pregnancy a 10-fold higher circulating output towards the feto-placental unit is achieved $[15,16]$ which can be seen by Doppler sonography and by calculating the Resistance Index (RI) or Pulsatility Index (PI) of the uterine arteries. Paralleling trophoblast invasion, most of the decrease in resistance of the uterine arteries is achieved until 22 weeks' gestation. The postsystolic notch disappears, paralleling the decrease in resistance until 22 weeks of gestation [6]. Failure of impaired interstitial trophoblast migration is associated with maintaining high uteroplacental vascular resistance [5]. Persisting elevated Resistance or Pulsatility Index of the uterine artery, especially at the placental uterine artery, indicates a significant elevated risk for fetal intrauterine growth restriction (IUGR) or the development of preeclampsia (PE) and, therefore, Doppler sonography is widely used to identify pregnant women with such elevated risk [2, 4, 5, 11-13]. Chronic reduction of uterine blood flow can also be observed in pregnancy-induced hypertension $(\mathrm{PIH})$ and diabetes mellitus in pregnancy [9].

Preeclampsia is a pregnancy associated disease with high resistance and vasospastic condition in contrast to the low resistance situation of normal pregnancies and it is defined as proteinuria $(0.3 \mathrm{~g} / \mathrm{L})$ accompanied by hypertension ( $\geq 140 / 90 \mathrm{mmHg}$ ) after 20 weeks of gestation. Intrauterine growth restriction is defined as fetal growth below the 5th centile. Pregnancy induced hypertension is characterized by high blood pressure detected for the first time after mid-pregnancy ( $\geq 140 / 90 \mathrm{mmHg})$ [14].

Fetal IUGR and maternal PE are the most common reasons for preterm delivery and preeclampsia is still one of the leading causes of maternal and fetal morbidity and mortality [21]. We previously demonstrated a systemic correlate showing a decrease of the $\mathrm{PI}$ at the maternal cubital artery paralleling advancing gestational age during the normal course of pregnancy [20]. The aim of the present study was to examine whether uterine and 
Table 1 Fetal outcome showing mean values, range and standard deviation (SD) of umbilical artery pH, Apgar Score at 5 and 10 min and fetal weight in the normal, PE and IUGR groups.

\begin{tabular}{llllll}
\hline Fetal outcome & $\begin{array}{l}\text { Week of gestation } \\
\text { at delivery } \\
\text { (range; SD) }\end{array}$ & $\begin{array}{l}\text { Fetal weight } \\
\text { grams } \\
\text { (range; SD) }\end{array}$ & $\begin{array}{l}\text { pH at umbilical } \\
\text { artery } \\
\text { (range; SD) }\end{array}$ & $\begin{array}{l}\text { APGAR-score } \\
\text { at 5 min } \\
\text { (range; SD) }\end{array}$ & $\begin{array}{l}\text { APGAR-score } \\
\text { at 10 min } \\
\text { (range; SD) }\end{array}$ \\
\hline Normal Pregnancies & $39.91(30.0-42 ; 0.91)$ & $3419(2171-4300 ; 471)$ & $7.33(7.13-7.43 ; 0.08)$ & $9.65(5-10 ; 0.95)$ & $9.71(8-10 ; 0.34)$ \\
Preeclampsia & $33.18(29.0-37.0 ; 0.83)$ & $1409(510-2350 ; 547)$ & $7.29(7.10-7.39 ; 0.06)$ & $9.36(8-10 ; 0.67)$ & $9.73(9-10 ; 0.47)$ \\
IUGR & $34.7(27.0-47.0 ; 1.14)$ & $1649(471-2270 ; 584)$ & $7.34(7.20-7.44 ; 0.06)$ & $9.7(9-10 ; 0.48)$ & $9.84(8-10 ; 0.32)$ \\
\hline
\end{tabular}

peripheral resistance in pregnancies complicated by fetal intrauterine growth restriction (IUGR) show elevated levels as one would expect in pregnancies complicated by preeclampsia or induced hypertension (PIH).

\section{Methods}

This was a prospective study, carried out in the first Obstetric and Gynecologic department of the Maximilians-University, Munich, from August 2001 to January 2004. The study included the following groups:

Normal pregnancies: Sixty-seven pregnancies with uneventful course of pregnancy were investigated in 4-6 week intervals. At each evaluation Doppler sonography was carried out on the placental, non-placental uterine and cubital artery. Fetal intrauterine growth restriction, maternal pregnancy induced hypertension or proteinuria, pathologic pregnancy outcome, maternal heart rate below 60 and above 100 beats per minute were exclusion criteria for the normal pregnancy group.

Preeclampsia: Seventeen patients with Preeclampsia (PE) were included to the study. Inclusion criteria for preeclampsia were blood pressure more than $140 / 100 \mathrm{mmHg}$ on two occasions and proteinuria more than $300 \mathrm{mg} / \mathrm{L}$ for the first time during pregnancy. Patients with a history of high blood pressure or renal disease were excluded. Doppler sonography was performed on the day of admission or diagnosis and was repeated according to clinical needs, at least every week.

Intrauterine growth restriction: Twelve patients with fetal intrauterine growth restriction (IUGR) meeting the criteria for the IUGR group (estimated fetal weight by ultrasound below the 5th centile with a postnatal birth weight below the 10th centile for gestational age) were included. All fetuses had abnormal umbilical Doppler with elevated PI. Patients with a history of hypertension or chronic medical disease were excluded as well as patients with newborns weighing $>$ the 10 centile. Doppler sonographic examination was performed on the day of admission or diagnosis and was repeated according to clinical needs, at least every week.

Doppler sonography: Investigations were performed on the left and right uterine artery-but were characterized by their relation to the placenta (placental and non-placental uterine artery) and on the cubital artery as previously described [20]. Briefly, Pulsed Doppler sonography was performed on a Logic 400 machine (GE, Kranzbühler, Solingen, Germany) with a $3.5 \mathrm{MHz}$ probe. For the maternal cubital artery, the angle of insonation was aimed at $<45^{\circ}$. For the measurements of the cubital artery, the probe was positioned on the relaxed lying right arm in the cubital area and the artery were visualized by color mode. The Doppler sample volume included the whole vessel diameter. Measurements were made when a continuous clear signal for the velocity wave form was available. The measurements were repeated twice and the lower resistance value of the PI was taken for analysis. The ultrasound machine and the $3.5 \mathrm{MHz}$ device underwent daily calibration. All examinations were conducted by the same experienced investigator (BS). The intra-observer variation coefficient was $6.96 \%$ for two fold repeated measurements $(n=14)$. From the analysis of the Doppler spectra the parameters maximum systolic velocity (Vmax), maximum end-diastolic velocity (Vmin) and mean velocity (Vmean) were derived. From these parameters the Pulsatility index (PI) was calculated: $\mathrm{PI}=(\mathrm{Vmax}-\mathrm{Vmin} /$ $V m e a n)$. As the patient is lying in recumbent-to-semi-recumbent left position, facing the investigator, the right cubital artery was chosen for convenience of the investigation. Great care was taken not to compress the cubital artery.

Statistical analysis was performed with the StatView Program (Spearmann-Rank-Correlation, Fisher's transformation, KruskalWallis-Test, Mann-Whitney $U$ test); a P-value $<0.05$ was considered significant.

The follow up during the study was in accordance with regular procedures in our department. The study was approved by the local University Ethics Committee (No. 158/00) and all patients gave written informed consent.

\section{Results}

Fetal outcome is summarized in Table 1. In normal pregnancies, a significant decrease at the uterine arteries $(\mathrm{P}<0.0001$ each), as well as at the cubital artery $(P<0.0001)$ was found. In normal pregnancies, the main decrease in uterine resistance happens until 22 weeks' gestation. To examine whether IUGR and PE pregnancies have elevated resistance levels we analyzed the PI at the uterine arteries and at the cubital artery from 22 weeks onwards until delivery.

Mean value and standard deviation of the PI at each the placental, non-placental and cubital artery of all measurements between 22 and 41 gestational weeks are summarized in Table 2. The mean $\mathrm{Pl}$ of measurements between 22 and 41 weeks at the cubital artery was 2.950 (range 1.125-12.710) in normal pregnancies, whereas it was elevated in the $\mathrm{PE}$ group (mean $\mathrm{PI} 3.777$; range 1.441-8.183) as well as in the IUGR group (mean PI 3.995; range 0.781-10.930). Similar results were found at the placental and non-placental uterine arteries showing higher resistance levels in the PE and IUGR group. 
Table 2 Mean values, range and standard deviation (SD) of the PI at the placental, non-placental uterine, and cubital arteries between 22 and 41 weeks' gestation in the normal pregnancy, PE and IUGR groups.

\begin{tabular}{lllll}
\hline & Group & Mean PI-value & Range & SD \\
\hline Placental uterine artery & normal & 0.678 & $0.371-1.254$ & 0.156 \\
Placental uterine artery & PE & 1.267 & $0.759-2.268$ & 0.431 \\
Placental uterine artery & IUGR & 1.063 & $0.498-2.61$ & 0.534 \\
Non-placental uterine artery & normal & 0.859 & $0.330-2.222$ & 0.289 \\
Non-placental uterine artery & PE & 1.631 & $0.881-2.741$ & 0.545 \\
Non-placental uterine artery & IUGR & 1.124 & $0.561-2.007$ & 0.439 \\
Cubital artery & normal & 2.950 & $1.125-12.710$ & 1.898 \\
Cubital artery & PE & 3.777 & $1.441-8.183$ & 1.965 \\
Cubital artery & IUGR & 3.995 & $0.781-10.930$ & 2.615 \\
\hline
\end{tabular}

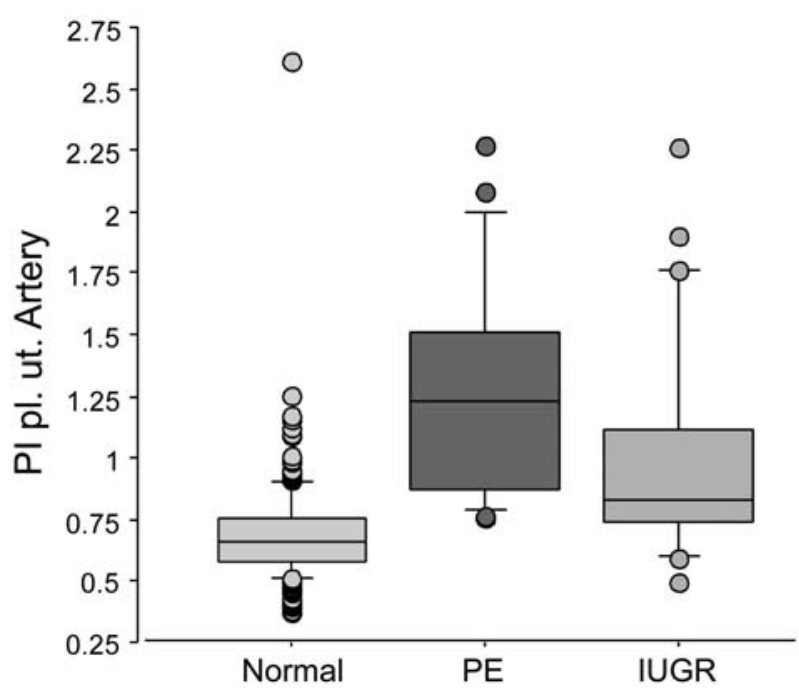

Figure 1 Box plots demonstrating the different values beyond 22 weeks of pregnancy at the placental uterine artery.

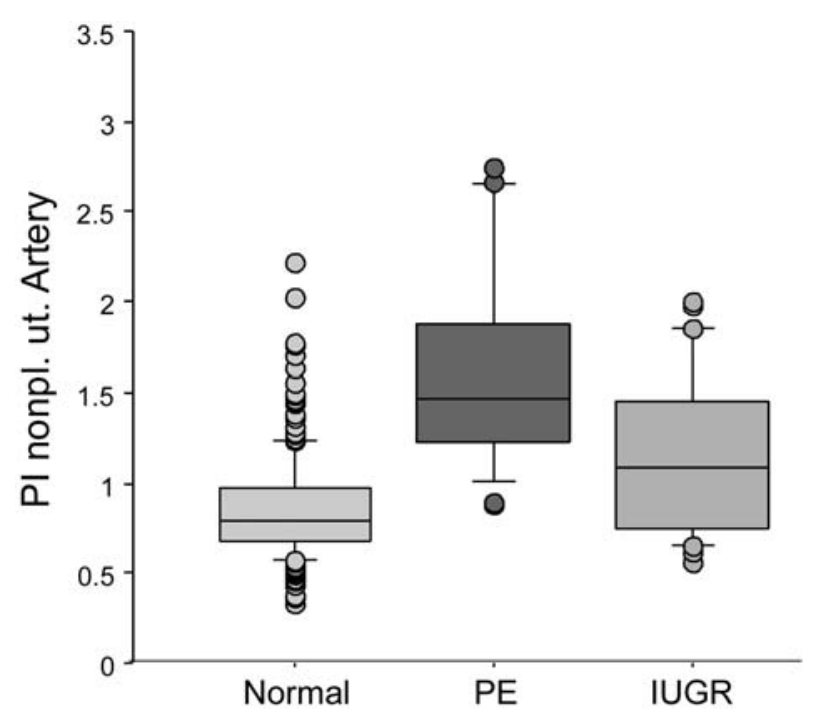

Figure 2 Box plots demonstrating the different values beyond 22 weeks of pregnancy at the non-placental uterine artery.
Statistical analysis of the means (Kruskal-Wallis test) revealed $\mathrm{P}$-values $<0.0001$ for the placental and nonplacental uterine arteries and a $\mathrm{P}$-value $<0.001$ for the cubital artery.

Comparing the pregnancy groups by each vessel, the following results were obtained (Mann-Whitney $U$ test): the mean $\mathrm{PI}$ at the placental uterine artery is significantly higher in the PE-group $(\mathrm{P}<0.0001)$ and in the IUGRgroup ( $\mathrm{P}<0.0001)$ compared to levels in the normal pregnancy group (Figure 1). The mean $\mathrm{PI}$ at the non-placental uterine artery is significantly higher in the PE-group $(P<0.0001)$ and in the IUGR-group $(P<0.005)$ (Figure 2$)$. Mean PI values of the cubital artery are significantly higher in the PE group $(P<0.01)$ and in the IUGR group $(P<0.05)$ compared to normal pregnancy values (Figure 3).

The differences between normal pregnancies and IUGR pregnancies, as well as compared to preeclamptic pregnancies, remain significant when only 1 measurement at admission is taken for analysis between 22 and 41 weeks: The mean PI at the placental uterine artery is

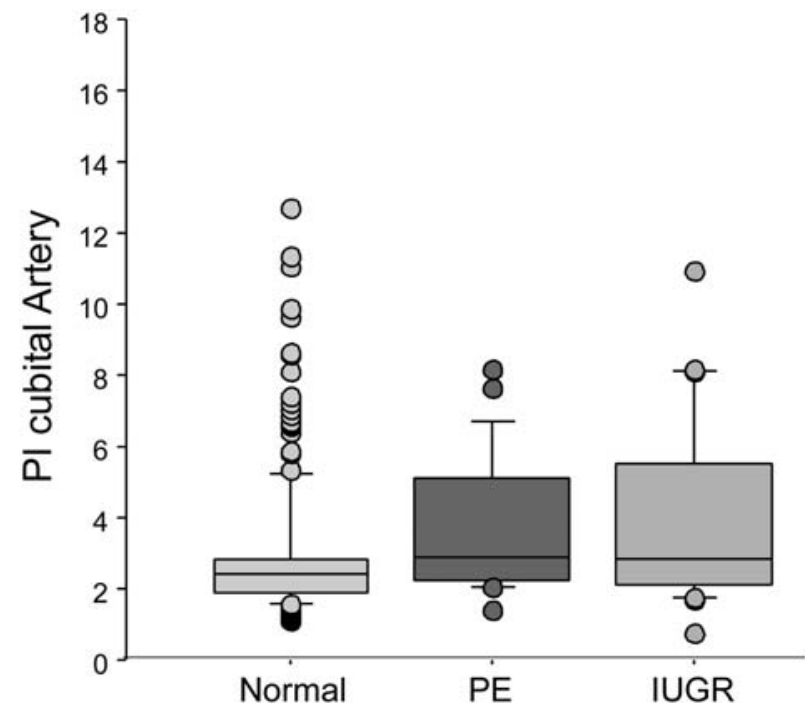

Figure 3 Box plots demonstrating the different values beyond 22 weeks of pregnancy at the cubital artery. 
0.774 (range 0.402-0.595) in the normal group and 1.055 in the IUGR group $(P<0.05)$ and 1.355 in the $P E$ group $(\mathrm{P}<0.001)$.

The mean PI at the non-placental artery is 0.952 (range 0.413-2.630) in the normal group and 1.269 (range $0.561-1.979)$ in the IUGR group $(P<0.05)$ and 1.872 in the PE group $(\mathrm{P}<0.001)$. The mean $\mathrm{PI}$ at the cubital artery is 3.005 (range 1.125-16.590) in the normal group and 4.652 in the IUGR group $(P<0.05)$ and 3.365 in the $P E$ group $(P<0.05)$.

A different "Doppler flow profile" can be noticed when different groups are compared: In normal pregnancy, no postystolic notching can be observed at the uterine artery after 22 weeks as well as no postsystolic notch in most $(91 \%)$ of the patients after 32 weeks at the cubital artery. In contrast, a postsystolic notch at the uterine artery in the presence of preeclampsia is observed, as well as notching or persistent postsystolic notch at the cubital artery (Figure 4).

\section{Discussion}

Normal pregnancy is associated with vasodilatation and decreased peripheral resistance [18]. General consent exists regarding preeclampsia as a systemic disease with profound systemic dysfunction of the maternal vascular endothelium $[10,17]$. Doppler sonography of the uterine arteries at 20-23 weeks has the capacity to predict a part of severe forms of adverse pregnancy outcome [1]. Takata et al. examined the ophthalmic arterial blood flow in women with preeclampsia and the flow mediated dilation at the brachial artery [22]. In preeclampsia, the flow mediated dilation at the brachial artery was lower, reflecting the impaired endothelial nitric oxide release after shear stress [22]. Interestingly, the PI at the maternal ophthalmic artery showed lowered resistance in patients affected by preeclampsia. The authors explain the latter observation as caused by an orbital hyperperfusion or hyperemia. Their results demonstrate that changes in endothelial function in preeclampsia vary in different organs.

Sagol et al. investigated the relationship between histopathological findings and uterine Doppler flow measurements at the uterine artery in patients with preeclampsia and fetal growth restriction: Patients with PE and fetal IUGR with elevated uterine resistance showed significant less invading trophoblast cells into the uterine myometrium upon histological examination. Their results support the hypothesis that high uterine artery flow resistance is related to a reduced or impaired trophoblast migration into the myometrium and inadequate physiological changes in the spiral arteries in women with the described pathologic courses of pregnancy [19].
Lin also examined placental bed biopsies taken at cesarean section and compared the findings to the uterine artery Doppler measurements before delivery. Those patients with impaired trophoblast migration had a higher incidence of small for gestational age infants $(P<0.01)$ and a higher systolic-diastolic ratio (S/D) measured at the uterine artery $(P<0.05)$. The authors concluded that abnormal uterine artery flow velocity waveforms may indicate impaired trophoblast migration into the myometrium at the placental bed.

Our results show a paralleling systemic vascular elevated resistance in pregnancies affected by preeclampsia, reflecting the pathogenesis of preeclampsia, defined as a high resistance vasospastic condition.

Several biochemical evidence demonstrate similar changes in preeclampsia and IUGR: Plasma levels of placental growth factor (PIGF) levels in pregnant women rise steadily throughout pregnancy from the level of nonpregnant women $(<50 \mathrm{pg} / \mathrm{mL}$ ) to levels exceeding $500 \mathrm{pg} / \mathrm{mL}$ after 30 weeks' gestation [7]. The rise in plasma PIGF in the second half of pregnancy was significantly attenuated in pregnancies complicated by preeclampsia in late gestation. Of all women who developed preeclampsia, $27.3 \%$ (12 of 44) had plasma PIGF levels below 200 pg/ $\mathrm{mL}$ [7]. Vascular endothelial growth factor is known to be altered in patients with preeclampsia: Kupferminc et al. demonstrated significantly increased plasma concentrations of VEGF in preeclamptic patients, compared with normal pregnancies (median, $47 \mathrm{ng} / \mathrm{mL}$; range, 10.6-72 $\mathrm{ng} / \mathrm{mL}$ versus median, $13.6 \mathrm{ng} / \mathrm{mL}$; range, $0.66-20 \mathrm{ng} / \mathrm{mL} ; \mathrm{P}<0.001$ ) [8]. Furthermore, the maternal plasma VEGF levels were correlated with the severity of hypertension, suggesting a role for VEGF in the pathogenesis of preeclampsia [8].

Our study demonstrates elevated resistance levels in the uterine as well as the peripheral cubital artery in the IUGR group similar to those seen in the preeclamptic group. Several studies demonstrated that children and young adults born after intrauterine growth restriction and preterm are at a higher cardiovascular risk, which can be demonstrated as an increase in systemic arterial stiffness and mean blood pressure [3]. So far, no data are published concerning the maternal systemic vascular resistance when fetal intrauterine growth restriction is present. We speculate whether maternal elevated resistance leads to an impaired blood flow and if this is accompanied by impaired or reduced trophoblast invasion and fetal growth restriction. This would be in accord with the findings of Sagol [19].

We conclude that measuring maternal arterial resistance by Doppler sonography in pregnancies complicated by impaired trophoblast invasion and uteroplacental circulation can show a vascular phenomenon paralleling the impaired uterine arteries transformation at the peripheral arteries. This finding is expected in preeclampsia but can also be seen in IUGR pregnancies. The authors 
A

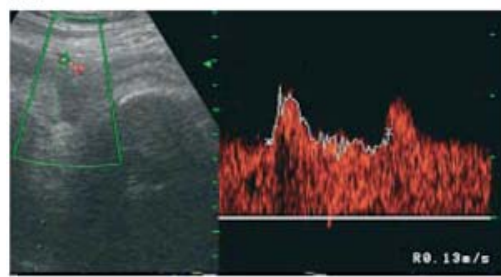

Plac. ut. artery, normal, 28 weeks

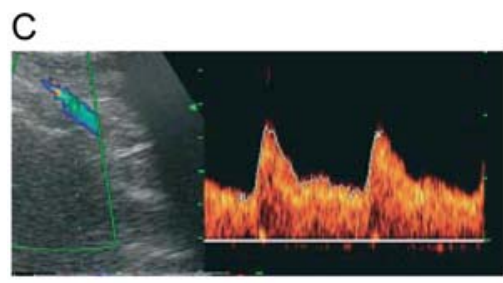

Cubital artery, normal, 34 weeks
B

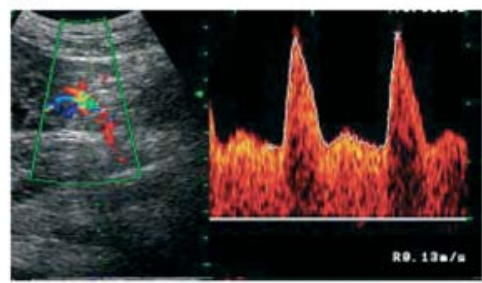

Plac. ut. artery, PE, 32 weeks

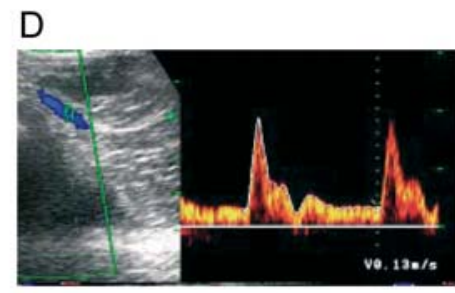

Cubital artery, PE, 32 weeks

Figure 4 Doppler profiles: Placental and cubital artery in normal $(A, C)$ and preeclamptic $(B, D)$ pregnancies.

acknowledge that the subjective observation of unchanged flow profiles at the cubital artery needs systematic evaluation, especially in IUGR. Our findings encourage further investigation of the maternal vascular and endothelial pathomechanism in pregnancies with IUGR.

\section{References}

[1] Becker R, R Vonk, W Vollert, M Entezami: Doppler sonography of uterine arteries at 20-23 weeks: risk assessment of adverse pregnancy outcome by quantification of impedance and notch. J Perinat Med 30 (2002) 388

[2] Bower S, K Schuchter, S Campbell: Doppler ultrasound screening as part of routine antenatal scanning: prediction of pre-eclampsia and intrauterine growth retardation. $\mathrm{Br} \mathrm{J}$ Obstet Gynaecol 100 (1993) 989

[3] Cheung YF, KY Wong, BC Lam, NS Tsoi: Relation of arterial stiffness with gestational age and birth weight. Arch Dis Child 89 (2004) 217

[4] Harrington K, D Cooper, C Lees, K Hecher, S Campbell: Doppler ultrasound of the uterine arteries: the importance of bilateral notching in the prediction of pre-eclampsia, placental abruption or delivery of a small-for-gestational-age baby. Ultrasound Obstet Gynecol 7 (1996) 182

[5] Kaufmann P, S Black, B Huppertz: Endovascular trophoblast invasion: implications for the pathogenesis of intrauterine growth retardation and preeclampsia. Biol Reprod 69 (2003) 1

[6] Kofinas AD, MA Espeland, M Penry, M Swain, CG Hatjis: Uteroplacental Doppler flow velocity waveform indices in normal pregnancy: a statistical exercise and the development of appropriate reference values. Am J Perinatol 9 (1992) 94

[7] Krauss T, HU Pauer, HG Augustin: Prospective analysis of placenta growth factor (PIGF) concentrations in the plasma of women with normal pregnancy and pregnancies complicated by preeclampsia. Hypertens Pregnancy 23 (2004) 101

[8] Kupferminc MJ, Y Daniel, T Englender et al.: Vascular endothelial growth factor is increased in patients with preeclampsia. Am J Reprod Immunol 38 (1997) 302

[9] Lang U, RS Baker, G Braems, M Zygmunt, W Kunzel, KE Clark: Uterine blood flow - a determinant of fetal growth. Eur J Obstet Gynecol Reprod Biol 110 (Suppl 1) (2003) S55

[10] McCarthy AL, RG Woolfson, SK Raju, L Poston: Abnormal endothelial cell function of resistance arteries from women with preeclampsia. Am J Obstet Gynecol 168 (1993) 1323

[11] Ohkuchi A, H Minakami, I Sato, H Mori, T Nakano, M Tateno: Predicting the risk of pre-eclampsia and a small-for-gestational-age infant by quantitative assessment of the diastolic notch in uterine artery flow velocity waveforms in unselected women. Ultrasound Obstet Gynecol 16 (2000) 171

[12] Papageorghiou AT, CK Yu, R Bindra, G Pandis, KH Nicolaides: Multicenter screening for pre-eclampsia and fetal growth restriction by transvaginal uterine artery Doppler at 23 weeks of gestation. Ultrasound Obstet Gynecol 18 (2001) 441

[13] Papageorghiou AT, CK Yu, S Cicero, S Bower, KH Nicolaides: Second-trimester uterine artery Doppler screening in unselected populations: a review. J Matern Fetal Neonatal Med 12 (2002) 78

[14] Peters RM, JM Flack: Hypertensive disorders of pregnancy. J Obstet Gynecol Neonatal Nurs 33 (2004) 209

[15] Pijnenborg R, JM Bland, WB Robertson, G Dixon, I Brosens: The pattern of interstitial trophoblastic invasion of the myometrium in early human pregnancy. Placenta 2 (1981) 303

[16] Pijnenborg R, G Dixon, WB Robertson, I Brosens: Trophoblastic invasion of human decidua from 8 to 18 weeks of pregnancy. Placenta 1 (1980) 3

[17] Roberts JM, CW Redman: Pre-eclampsia: more than pregnancy-induced hypertension. Lancet 341 (1993) 1447

[18] Robson SC, S Hunter, RJ Boys, W Dunlop: Serial study of 
factors influencing changes in cardiac output during human pregnancy. Am J Physiol 256 (1989) H1060

[19] Sagol S, E Ozkinay, K Oztekin, N Ozdemir: The comparison of uterine artery Doppler velocimetry with the histopathology of the placental bed. Aust N Z J Obstet Gynaecol 39 (1999) 324

[20] SchiessI B, CJ Strasburger, M Spannagl, F Kainer: Decreasing peripheral resistance during pregnancy monitored at the cubital artery. Eur J Clin Invest 33 (2003) 346
[21] Schlembach D: Pre-eclampsia - still a disease of theories. Fukushima J Med Sci 49 (2003) 69

[22] Takata M, M Nakatsuka, T Kudo: Differential blood flow in uterine, ophthalmic, and brachial arteries of preeclamptic women. Obstet Gynecol 100 (2002) 931

Received April 12, 2005. Revised September 12, 2005. Accepted September 19, 2005. 